\title{
Construir 34.00o camas hospitalarias en España. El reto del Plan Nacional de Instalaciones Sanitarias (1942-1982).
}

\section{Build 34,0oo hospital beds in Spain. The challenge of the National Plan of Sani- tary Installations (1942-1982).}

\author{
Alba Lorente de Diego ${ }^{(*)}$, César Martín-Gómez ${ }^{(* *)}$, Francisco Javier Castro Molina ${ }^{(* * *)}$
}

\section{RESUMEN}

En un periodo marcado por la escasez y las dificultades económicas, se apostó en España por la realización de un gran plan sanitario que afectaría a toda geografía española, llamado el Plan de Instalaciones Sanitarias. Promovido por el propio Gobierno de la época, se propuso la construcción de una red sanitaria fundamentada en la construcción de hospitales y ambulatorios, a imagen y semejanza de la modernidad de los edificios americanos. Se construyeron más de un centenar de grandes complejos hospitalarios de forma rápida, pero a la vez insuficiente. En menos de una década, una treintena de nuevos hospitales y ambulatorios formaban parte del conjunto urbano de las ciudades españolas. El desarrollo del plan duró cuatro décadas, el cual estuvo marcado por la inestabilidad en la ejecución de los proyectos, y por el esfuerzo técnico, económico y político que conllevó.

Palabras clave: asistencia; siglo XX; sanidad; hospital en bloque; Instituto Nacional de Previsión; residencia sanitaria.

\section{ABSTRACT}

In a period marked by scarcity and economic difficulties, Spain opted for the realization of a large health plan that would affect every Spanish geography, called the Health Facilities Plan. Promoted by the Government of the time, the construction of a health network based on the construction of hospitals and clinics was proposed, in the image and likeness of the modernity of American buildings. More than a hundred large hospital complexes were built quickly, but at the same time was insufficient. In less than a decade, about thirty new hospitals and clinics were already part of the urban complex of Spanish cities. The development of the plan lasted four decades, which was marked by instability in the execution of the projects, and by the technical, economic and political effort involved.

Keywords: assistance; twentieth century; health; block hospital; National Institute of Forecast; sanitary residence.

$\left({ }^{*}\right)$ Arquitecta. Universidad de Navarra, Pamplona (España).

(**) Doctor Arquitecto. Departamento de Construcción, Instalaciones y Estructuras. Universidad de Navarra, Pamplona (España).. $\left({ }^{* * *}\right)$ Doctor en Historia del Arte. Investigador y profesor en la Escuela de Enfermería de Nuestra Señora de Candelaria, Universidad de La Laguna, Tenerife (España).

Persona de contacto/Corresponding author: alorente.1@alumni.unav.es (A. Lorente de Diego)

ORCID: http://orcid.org/oooo-0oo3-2765-5720 (A. Lorente); http://orcid.org/oooo-0oo3-0150-1686 (C. Martín-Gómez); https://orcid.org/oooo-0001-6068-9178 (F. Castro Molina)

Cómo citar este artículo/Citation: Alba Lorente de Diego, César Martín-Gómez, Francisco Javier Castro Molina (2021). Construir 34.00o camas hospitalarias en España. El reto del Plan Nacional de Instalaciones Sanitarias (1942-1982). Informes de la Construcción, 73(562): e385. https:// doi.org/10.3989/ic.78487

Copyright: (C) 2021 CSIC. Este es un artículo de acceso abierto distribuido bajo los términos de la licencia de uso y distribución Creative Commons Reconocimiento 4.0 Internacional (CC BY 4.0). 


\section{INTRODUCCIÓN}

Durante las primeras décadas del siglo XX coexistieron en España distintas corrientes arquitectónicas que pocas veces se presentaron en su forma más pura, sino más bien como síntesis de todas ellas. El modernismo y el nacionalismo predominaban, pero se advirtieron síntomas de la llegada de corrientes nuevas como el movimiento moderno; y tipologías como la arquitectura hospitalaria se «contagiaron" de la llegada de estas ideas, dando lugar a un periodo de transición donde lo tradicional y moderno se mezclaron.

La sanidad española sufría de escasez de recursos, el reflejo más claro de esta realidad fue el escaso número de hospitales que había en todo el país. Para subsanar esta situación, se promulgaron una serie de leyes sanitarias y se impulsó la construcción de hospitales, pero la llegada de la Guerra Civil interrumpió el camino hacia el progreso, y la modernización iniciada fundamentalmente a partir de los años veinte.

Después de la Guerra, la situación no fue mejor. El país se encontraba en una profunda crisis económica y social, fruto de las heridas causadas por la guerra. Aun así, se retomó la idea subsanar las carencias sanitarias del país, creando el Plan Nacional de Instalaciones Sanitarias, cuyo objetivo fue la creación de un importante número de hospitales y ambulatorios por toda la geografía española. Llevados a cabo por el Instituto Nacional de Previsión (INP), los nuevos hospitales, o residencias sanitarias, se proyectaron a imagen y semejanza de las grandes construcciones americanas, símbolo de modernidad y progreso. Hecho paradójico si se coloca en paralelo con la vuelta a las «esencias históricas de la nación» (1) que promovía el régimen.

Tras esta exposición del contexto, el objetivo del artículo es analizar el Plan de Instalaciones Sanitarias, desde el porqué de su nacimiento analizando los antecedentes previos, su desarrollo y final. Para ello se va a centrar la atención en las construcciones más destacadas buscando entender su desarrollo a través de las variaciones constructivas que se fueron dando con el paso tiempo.

\section{ANTES DEL PLAN}

Desde principios del siglo $\mathrm{XX}$ el número de instituciones públicas para la atención de enfermos era escasa (tabla 1), aunque había una tendencia a la mejora en la asistencia gratuita a enfermos (2). Aun así la beneficencia era un servicio limitado, no accesible para todos, pues según lo definía la Ley, la beneficencia era un conjunto de prestaciones que el Estado y particulares ofrecían a aquellos incapaces de procurarse la subsistencia, y prohibía de manera expresa, tanto a los establecimientos de beneficencia privada como pública, admitir a pobres o mendigos válidos para el trabajo (2). Esta definición dividía a la sociedad en dos grupos: aquellos que podían pagarse una asistencia privada; y los mendigos o inválidos. La clase media era la más perjudicada, al ser una parte de la sociedad dependiente del salario (generalmente bajo) y afectada por nuevos problemas de salud fruto del hacinamiento y la mala higiene de las ciudades industriales que estaban surgiendo.

Es entonces cuando se planteó la necesidad de definir una política hospitalaria que mitigara la ínfima calidad sanitaria del país (3). Los primeros cambios se dieron en el ámbito legislativo, poniéndose en marcha un conjunto de medidas que supusieron el inicio y desarrollo de la sanidad pública en España. El 22 de enero de 1904 se creó la Instrucción General de Sanidad, que, sin tener el rango de Ley, a efectos prácticos funcionó como tal. Tuvo la función de estructurar la administración en tres campos: ejecutivo, consultivo e inspector (4). Otra de las iniciativas legislativas fue la creación en 1908 del Instituto Nacional de Previsión de la mano de Antonio Maura y Alfonso XIII, que tuvo un papel esencial en la implantación de los seguros sociales y la planificación de futuros hospitales por toda la geografía española.

Tabla 1. Establecimientos de beneficencia general en España en 1909.

\begin{tabular}{|l|l|l|l|}
\hline Nombre & Localidad & Especialidad & \\
\hline o1 & Hospital de la Princesa & Madrid & Afecciones agudas no infecciosas. Ambos sexos. \\
\hline 02 & Hospital de Jesús Nazareno & Madrid & Mujeres Impedidas e incurables \\
\hline 03 & Hospital de Nuestra Señora del Carmen & Madrid & Mujeres Impedidas e incurables \\
\hline o4 & Hospital de Toledo & Toledo & Decrépitos y ciegos de ambos sexos \\
\hline o5 & Hospital Manicomio Santa Isabel & Leganés & Dementes de ambos sexos \\
\hline o6 & Hospital Hidrológico de Carlos III & Guadalajara & Asistencia a enfermos \\
\hline o7 & Colegio de Santa Catalina de los Donados & Madrid & Refugio de ciegos menores \\
\hline o8 & Colegio de Huérfanas La Unión & Aranjuez & Atención a huérfanas menores \\
\hline o9 & Patronato Nacional de las Hurdes & Cáceres & Remediar las necesidades de la región \\
\hline 10 & Instituto Oftalmológico Nacional & Madrid & Asistencia a enfermos \\
\hline 11 & Instituto de Inválidos del Trabajo & Madrid & Reeducación profesional \\
\hline 12 & Manicomio de Nuestra Señora del Pilar & Zaragoza & Dementes \\
\hline 13 & Asilos de San Juan y Santa María de El Pardo & Madrid & Asilo de niños y niñas \\
\hline 14 & Asilo de Hijos de Lavanderas & Madrid & Asilo de menores \\
\hline 15 & Patronato Nacional de Protección de Ciegos & Madrid & Educación de ciegos \\
\hline
\end{tabular}

Fuente: Pons Pons, J., \& Vilar Rodíguez, M. (2015). El Seguro de Salud Privado y Público en España: Su análisis en perspectiva histórica (1880-2013). 
Respecto a los avances en arquitectura hospitalaria, uno de los últimos ejemplos hospitalarios españoles de tipología pabellonar fue el Hospital de la Santa Cruz y San Pablo de Barcelona. Diseñado por Lluís Domènech en 1902, y finalizado en 1930, proyectó un edificio de concepto paisajístico compuesto por edificios independientes de planta rectangular rodeados de amplios jardines, los que enlazó por un entramado de galerías subterráneas con la finalidad de hacer al conjunto más funcional. Estos nuevos hospitales de tipología pabellonar evidenciaron un cambio a la hora de acometer los proyectos hospitalarios. Se comenzó a tener en cuenta aspectos presupuestarios, de gestión y de funcionamiento; y a tener gran importancia el soleamiento, los materiales constructivos, la organización de las distintas áreas del hospital y la ventilación de los espacios, que, junto con los avances en la tecnología de sistemas de calefacción y ventilación, cambiaron la forma de planificar los hospitales.

Un ejemplo de que la tipología de hospital estaba cambiando fue el Hospital de San Francisco de Paula u Hospital de Jornaleros de Madrid, construido en 1908 por Antonio Palacios y Joaquín Otamendi (5). Proyectaron un hospital que se alejaba de la tipología pabellonar, dibujando más una construcción tipo monumental. La planta (figura 1) era en forma estrellada, lo que recuerda al proyecto de Antoine Petit para el Hôtel Dieu de París de 1774 (6) resultado de la suma de varios pabellones articulados en su centro.

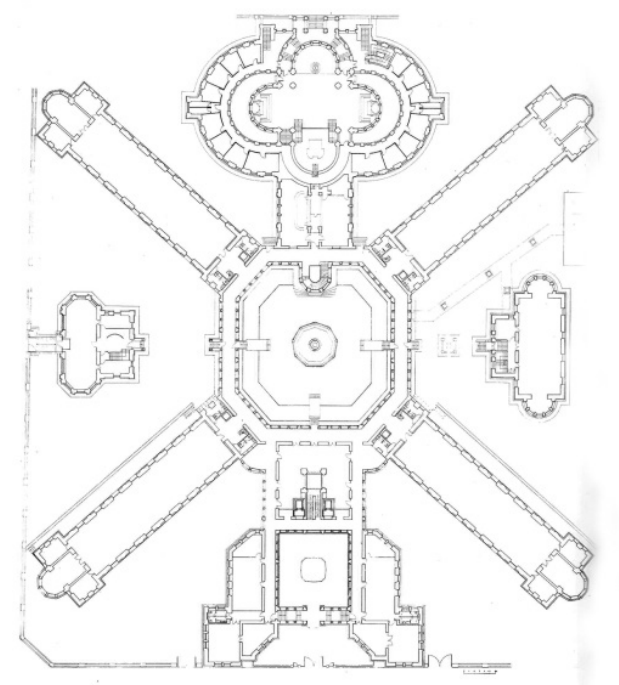

Figura 1. Plano de la planta baja del Hospital de San Francisco de Paula en Madrid, 1908.

Fuente: Pieltaín ÁIvarez-Arenas, A. (2003). Los hospitales de Franco : la versión autóctona de una arquitectura moderna (Universidad Politécnica de Madrid - Escuela Técnica Superior de Arquitectura)

\section{3. ¿HOSPITAL DE TIPOLOGÍA PABELLONAR O TI- POLOGÍA EN BLOQUE?}

Este debate dejó de producirse por los especialistas a principios del siglo XX (7), y la respuesta fue el desarrollo del hospital en bloque. Las diferencias que equilibraron la balanza a favor del hospital en bloque, fueron fundamentalmente la disminución de recorridos al compactar los servicios y poder superponerlos en altura, gracias a la invención de sistemas como el ascensor; todo ello acompañado del consiguiente ahorro en climatización, alumbrado y en general, de todos los gastos de mantenimiento. Además, el hospital de tipología pabellonar dejó de tener sentido, pues estaba basado en la teoría miasmática, la cual fue superada por la teoría de los gérmenes.

Las primeras construcciones de este tipo se dieron en Estados Unidos. Los hospitales construidos en el país americano, tenían entre 5 ó 6 alturas, pero en 1915 el Fith Avenue Hospital de Nueva York ya contó con 11 alturas (8). Las plantas de estos nuevos hospitales se organizaban en forma de $\mathrm{T}, \mathrm{H}$ o Y, superponiendo verticalmente las especialidades clínicas y centralizando sus servicios en la planta baja del edificio (3). En cada planta se distribuía cada departamento médico completo, y en función del tipo formal, la planta contaba con uno o varios núcleos de comunicación vertical (8). Como referente del modelo americano destacó el Columbia Presbyterian Medical Center de Nueva York, construido en 1928 por Jambes Gamble Rogers, el cual contaba con 22 plantas.

En España, frente a las grandes construcciones americanas, nos encontramos con edificios más modestos como el Hospital de Toledo de 1926, o el Hospital Clínico San Carlos de Madrid de 1928, ambos de Manuel Sánchez Arcas. En uno y otro edificio nos hallamos con una nueva forma de entender el hospital, basada en la comunicación vertical versus a la horizontal.

El Hospital de Toledo, construido por Manuel Sánchez Arcas, Luis Lacasa y Francisco Solana en 1926, fue resultado de un concurso donde las bases traslucían una preferencia hacia la tipología pabellonar. Sin embargo, entre todas las propuestas presentadas, la de Sánchez Arcas, Lacasa y Solana, destacó por presentar un hospital más unitario y «moderno». El hospital se articuló en dos partes: servicios y hospital. Esta sectorización se vio favorecida por salvar el desnivel del terreno donde se localizó el proyecto, una colina junto a la línea de fe-

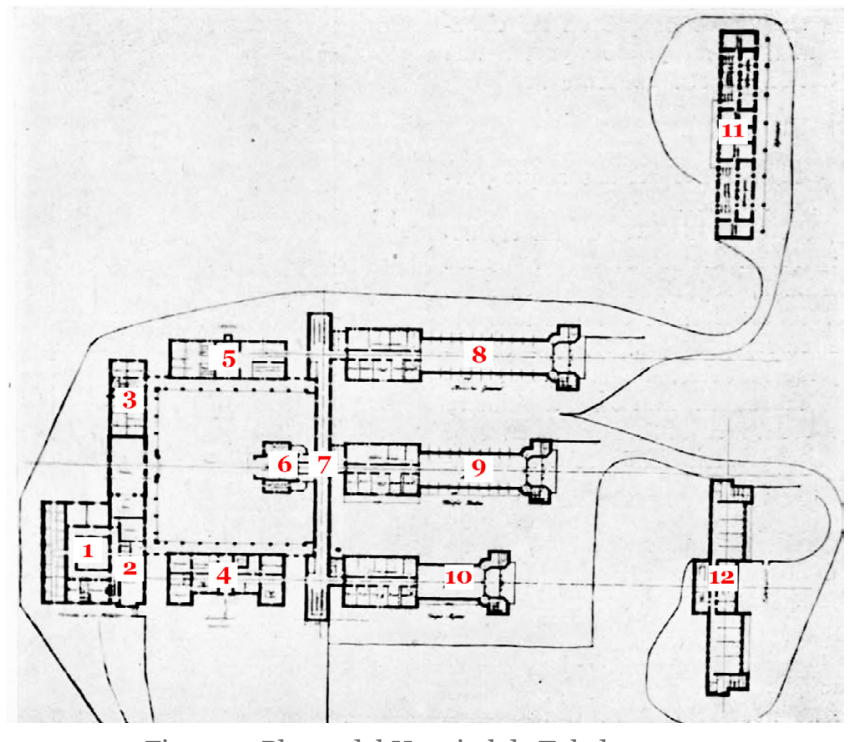

Figura 2. Planta del Hospital de Toledo, 1930.

Disposición: 1 -Convento; 2 - Capilla; 3 - Capellanes; 4 - Administración y consultas; 5 - Quirófano; 6 - Cocina y Servicios; 7 - Galería; 8 - Medicina general; 9 - Cirugía hombres; 10 - Cirugía mujeres; 11 Infecciosos; 12 - Tuberculosos; 13 - Autopsias y depósito de cadáveres.

Fuente: Sánchez Arcas, M., Luis, L., \& Francisco, S. (1931). Nuevo hospital provincial en Toledo. Arquitectura, (147), 226-233. 
rrocarril de Toledo. Esta fragmentación en diversos edificios desdibujó la idea de la tipología en bloque. La planta (figura 2) dibujaba esta sectorización con una parte de pabellones en forma de «peine», unidos entre sí por una galería transversal que a su vez los unía con la otra parte del hospital. Los pabellones, situados en la parte más alta, es donde se dispusieron las secciones de medicina general, cirugía para hombres y cirugía para mujeres. En la otra parte del edificio, situada en la zona más baja, se dispusieron la administración, consultas, cocinas y servicios. La construcción en ladrillo adquiere unos valores plásticos que no desentonaron con la arquitectura popular de Toledo, conjugando modernidad y tradición.

Otro de los grandes ejemplos de arquitectura hospitalaria en España, es el Hospital Clínico San Carlos de Madrid, diseñado por Manuel Sánchez Arcas en colaboración con Eduardo Torroja. Proyectado en 1928, diseñaron un conjunto de trazado regular y simétrico, reflejando la modernidad en los volúmenes puros de ladrillo cara vista, dónde sólo las losas de los soláriums aportaron un punto de expresividad ante la desnudez formal del edificio.

Este esquema dio lugar a una clínica por planta, desarrollada en dos bloques principales estrechos y alargados, situando en ellos los servicios universitarios y clínicos; más seis habitaciones para los enfermos con cuatro camas cada una. Ortogonalmente, en la fachada suroeste, se ubicaron cuatro bloques de enfermerías con una capacidad para 72 camas, y otros cuatro bloques en la fachada noreste. Paralelamente a este bloque se situó otro con idéntica distribución, el cual forma otra clínica. Estos se unían por el noreste con otro bloque en forma de «I», dividido en dos partes: dotaciones de enseñanza e investigación. El conjunto encerraba un patio, que, bajo éste en el sótano, se ubican los servicios. Además, en las esquinas se adosan cuatro piezas para alojar las cátedras quirúrgicas. El Hospital Clínico San Carlos fue un claro ejemplo de la asimilación de las nuevas ideas, con una organización típica en peine, diseñado a imagen y semejanza de los americanos (figura 3), adecuándolo a las necesidades propias del hospital. Al igual que en los ejemplos americanos, colocó las enfermerías en piezas perpendiculares al cuerpo principal, terminándolas con una terraza a modo de solárium.

Frente a la continuidad del antiguo modelo hospitalario, la defensa de la tipología en bloque la encontramos en un artículo publicado en la revista Arquitectura escrito por Manuel Sánchez Arcas en 1929. En él describió el Columbia Presbyterian Medical Center de Nueva York, destacando la funcionalidad, la disposición clara de las áreas y la buena resolución de las circulaciones, que los hospitales en bloque aportaban. Así lo ejemplificó al final del artículo:

«Todos estos problemas aunados están resueltos en este edificio, que es una prueba de la gran contribución que a los problemas de la arquitectura de nuestra época aportan los grandes hospitales modernos, de que tan brillante ejemplo es el Centro Médico (9).»

En la década de los treinta, hasta el comienzo de Guerra Civil (1936), surgieron proyectos hospitalarios que no pasaron del papel, pero igualmente interesantes. Tal y como muestran los archivos de la revista Arquitectura se realizaron los proyectos para un hospital en Logroño, Granada y San Sebastián. El proyecto (no construido) para el Hospital de Logroño fue
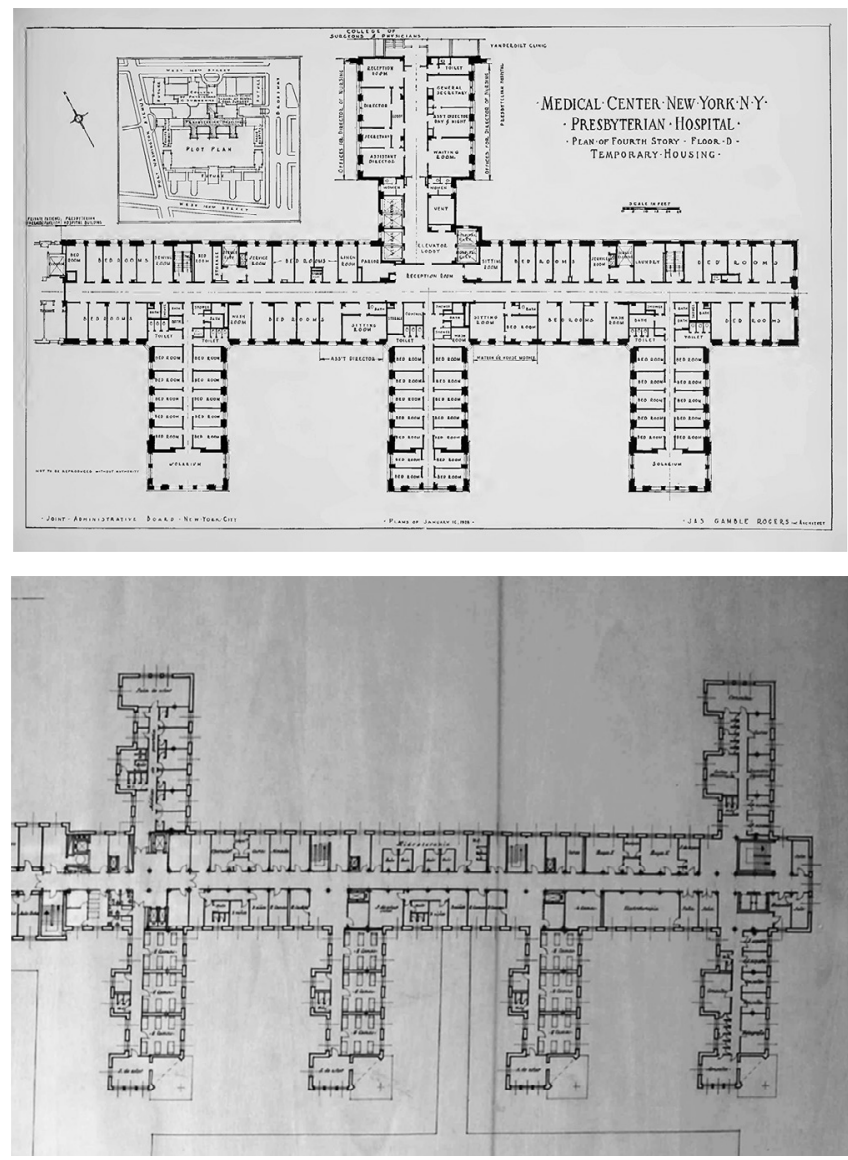

Figura 3. Comparación de la similitud existente entre las alas de enfermería del Hospital Presbiteriano de Nueva York (superior) y del Hospital Clínico San Carlos de Madrid (inferior), ambos de 1928.

Fuente: Elaboración Propia.

proyectado por Manuel Sánchez Arcas y Luis Lacasa. Fue fruto de un concurso en el año 1929, en el que ganaron el primer premio. La propuesta consistió en un edificio de arquitectura racional distribuido en tres agrupaciones de edificios. La primera agrupó a los servicios de administración, portería, etcétera; la segunda correspondió al servicio de hospitalización; y la tercera acogía a los tuberculosos e infecciosos. Centrándonos en el edificio de carácter hospitalario, su planta (figura 4) estaba configurada en forma de peine, con un volumen alargado central (como si fuera un gran pasillo distribuidor) que da acceso al resto de alas del hospital. No tenía gran altura, cuatro plantas, pero en el anteproyecto presentado, los arquitectos justificaron esta solución de altura como un ejemplo de modernidad, al no ceñirse al modelo constructivo de una planta que tanto recuerda a la tipología pabellonar:

«El edificio no puede ser de una planta, porque está claramente estudiado que un hospital cuyo número de camas sea superior a 80, y más teniendo distintas especialidades, cuyos servicios han de ser muy complejos, es antieconómico en todos los aspectos. [...] Si un hospital moderno tiene más de una planta, es imprescindible el montacargas para el traslado de enfermos y servicio de cocinas, [...] En el caso que nos ocupa, nos parece indicada la solución de un hospital con cuatro plantas de enfermerías por las razones siguientes: $1^{\mathrm{a}}$. Ventaja económica $[\ldots] 2^{\mathrm{a}}$. Ventada de esta concentración es la de acortar las distancias horizontales, (10)» 


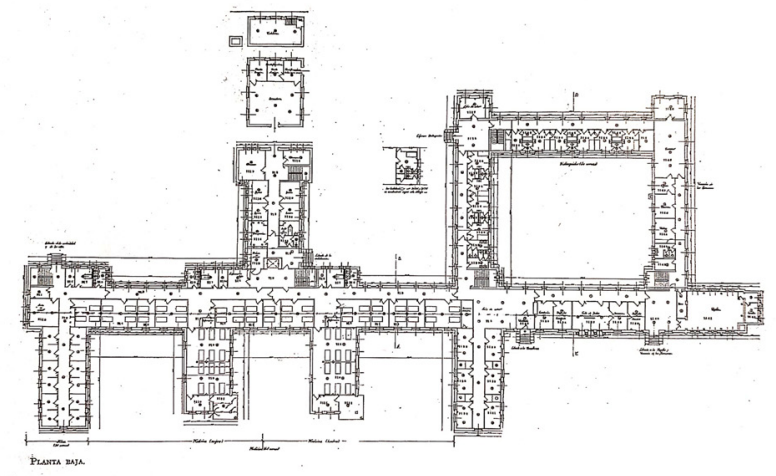

Figura 4. Planta del Hospital de Logroño. Manuel Sánchez Arcas, 1929.

Fuente: VV.AA. (2003). Manuel Sánchez Arcas, arquitecto (Fundación Arquia, ed.). Madrid.

El proyecto y construcción del hospital de Granada, igualmente surgió a raíz de un concurso realizado en $1927 . \mathrm{Ga}-$ nado por los arquitectos Aurelio Botella y Sebastián Vilata, el hospital se organizó entorno a un gran patio central en dos niveles según cuatro conjuntos simétricos destinados cada uno de ellos a un servicio médico. La planta (figura 5) no siguió los cánones iniciados de tipología en bloque y configuración en forma de "peine», teniendo una ordenación más clásica. Aun así, se puede adscribir dentro de la arquitectura racionalista y funcionalista europea, con una volumetría blanca, sin ornamentaciones, rotunda y escalonada en distintos niveles. Sufrió un gran deterioro durante la Guerra Civil por su empleo como hospital de campaña. Finalmente fue restaurado y reinaugurado en el año 1953 (11).

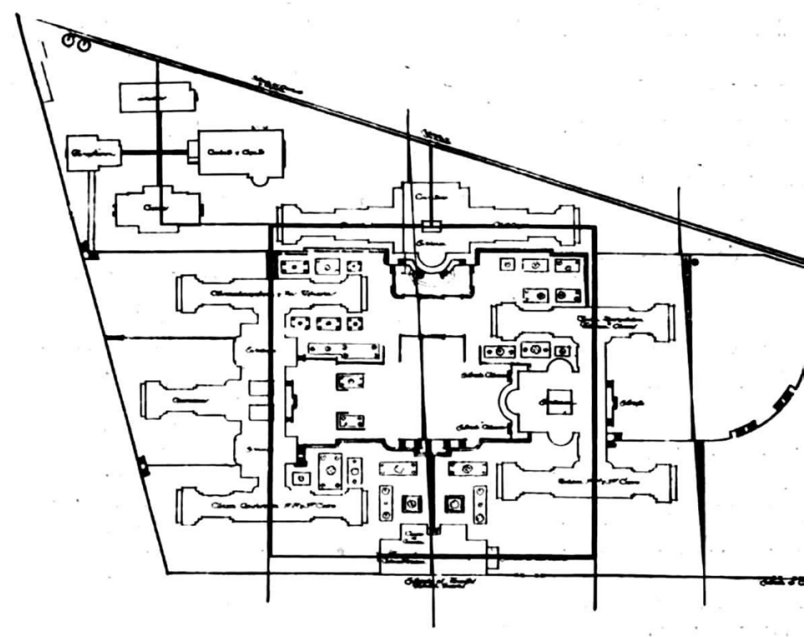

Figura 5. Planta del Hospital de Granada, 1927.

Fuente: Vilata Valls, S., Aurelio, B., de Cotes, L., \& de, S. L. (1931). Concurso para Facultad de Medicina y Hospital Clínico en Granada. Arquitectura, (143), 88-109.

Uno de los últimos proyectos hospitalarios de gran envergadura en España realizados antes del comienzo de la Guerra Civil, fue el proyecto para el Hospital de San Sebastián. Convocado a través de concurso, participaron
21 equipos, de los que ganó el formado por Francisco de Arcola y Domingo Aguirrebengoa. El proyecto presentado siguió los criterios de la moderna arquitectura hospitalaria, a imagen de los hospitales norteamericanos, y así lo reconocen en la memoria. La planta en forma de "peine», se distribuyó a través de dos cuerpos centrales alargados a los que perpendicularmente se dio acceso a las enfermerías. En la memoria del anteproyecto hicieron una clara defensa del hospital como una "máquina de sanación», un objeto arquitectónico que deja de ser un mero contenedor de espacios y personas, para convertirse en un objeto útil capaz de transformarse a las necesidades del usuario y de las funciones que en él se realizan. Sus palabras fueron las siguientes:

\section{«Principios básicos del proyecto}

En nuestro estudio hemos seguido unas cuantas normas generales impuestas en parte por las bases del concurso, en parte por la limitación económica y, en parte finalmente, por no apartarnos de nuestra concepción de lo que debe ser un hospital.

$1^{\mathrm{o}}$. El hospital es un instrumento de trabajo. No se puede concebirlo más que prestando el máximun de rendimiento. No se debe desperdiciar el espacio ni el dinero en fines suntuarios.

$2^{\circ}$. Más importancia que el estudio del edificio, pudiéramos decir que tiene el de su funcionamiento: el hospital es una institución viva y no se puede iniciar el estudio de su proyecto sin conocer profundamente la mecánica del funcionamiento hospitalario.

$3^{\circ}$. Es difícil proyectar un hospital sin conocer cuánto ha de costar su sostenimiento. La solución que se puede dar a muchos problemas, depende muy especialmente del problema del sostenimiento. Aunque no se conoce éste, hemos procurado siempre acudir a las soluciones del sostenimiento más económico.

$4^{0}$ Se inicia una era en la que ha de encarecerse extraordinariamente el coste del personal auxiliar y, por lo tanto, tendremos en nuestro anteproyecto a su limitación y obtención del máximun de eficiencia. (12)»

\section{PLAN NACIONAL DE INSTALACIONES SANITA- RIAS}

El Plan Nacional de Instalaciones estuvo vigente durante 35 años, y en ese tiempo su desarrollo estuvo marcado por diferentes episodios, pudiéndose diferenciar tres etapas principales. La primera comenzó con la puesta en marcha del Plan y la ejecución de los primeros objetivos, hasta la llegada de un periodo de estancamiento, dando inicio a la segunda etapa, marcada por el esfuerzo de construir los proyectos diseñados anteriormente y superar los problemas financieros. La tercera y última de las etapas, comenzó en un año singular, el vigésimo quinto aniversario del fin de la guerra, que bajo la idea de «grandiosidad» trajo nuevas formas y configuraciones hospitalarias. 


\subsection{Primera etapa (1942-1957)}

El primer precedente legal del Plan Nacional de Instalaciones Sanitarias apareció por primera vez en la Ley del 14 de diciembre de 1942, por la cual se creó el Seguro Obligatorio de Enfermedad y se concedió al Instituto Nacional de Previsión (INP) un plazo de seis meses para formular un reglamento y un estudio para llevar a cabo dicho plan (2). Los encargados de realizar dicho estudio fueron Primitivo Quintana López y Joaquín Espinosa Ferrándiz (13). En el informe presentado por Quintana López, Plan General de asistencia médica y de medicina preventiva para la implantación del seguro de enfermedad en España, proponía la creación de 34.000 nuevas camas, distribuidas entre tres tipos de construcciones hospitalarias: grandes y medianas instalaciones, y clínicas rurales; siguiendo el tipo de construcción vertical y en bloque como los hospitales norteamericanos modelo monoblock, o el Hospital Beaujon, de Paris (13).

Para denominar a las nuevas instalaciones sanitarias, los encargados del Plan descartaron los términos hospital y dispensario, pues tanto uno como el otro, se habían asociado siempre a estructuras de la beneficencia, poco eficaces en su misión. Por ello, en vez de utilizar esos términos comunes, eligieron otros que aludían al régimen ambulatorio o al de ingreso de beneficiarios en las instituciones; y así nacieron los ambulatorios y residencias sanitarias del Seguro Obrero de Enfermedad (14).

El 19 de febrero de 1945 finalmente se aprobó la Orden que dio inicio al Plan Nacional de Instalaciones Sanitarias, en el cual se proyectaba la construcción de 86 residencias y 149 ambulatorios (14); aunque no se dio el inicio para la construcción de las residencias y ambulatorios previstos. En primer lugar, en enero de 1946 varios miembros del departamento de arquitectura del Plan, Juan Pedro de la Cámara, Shaw Loring y Eduardo de Garay; emprendieron un viaje a Estados Unidos con el fin estudiar los hospitales y ambulatorias de aquel país. Visitaron hospitales de Nueva York, Washington y Baltimore (15).

A su llegada Eduardo de Garay publicó el libro: Los hospitales en Estados Unidos. Notas de un viaje de estudio, donde analizó las características más arquitectónicas de los hospitales, dejando el estudio funcional de las manos de su compañero De la Cámara. En el libro Garay realizó un estudio por partes, tanto gráfica como escrita, de los hospitales visitados, con atención a partes esenciales como a la calefacción, climatización, alumbrado o decoración. Los hospitales analizados fueron de tipo bloque y en altura, con plantas distribuidas a través de un cuerpo principal y otros anexos perpendicularmente donde se disponen las salas de enfermería. Ejemplo de ello son el New York Hospital y el Cornell University Medical College, ambos en Nueva York. Otros ejemplos analizados fueron el Hospital Médico Quirúrgico de Nueva York con una planta abierta en forma de C; y el Wesley Hospital de Chicago, un hospital de tipología de torre y basamento, con una planta en forma de X, que se salía de los esquemas tradicionales. Llegó a la conclusión de que la arquitectura hospitalaria americana se basaba en un funcionalismo fundamentado en el programa de necesidades, y en los nuevos materiales y técnicas constructivas, con una imagen moderna (15).

Después de estudiar los ejemplos americanos, el siguiente paso fue convocar en marzo de 1946 un Concurso de Ante- proyectos de Residencias Sanitarias y Ambulatorios, en el que se debía proyectar una residencia sanitaria para 500 camas, otra de 100 camas y un ambulatorio completo. Los ganadores fueron los arquitectos Aurelio Botella Enríquez y Sebastián Vilata Valls, para la residencia de 500 camas (figura 6); los arquitectos Manuel Martínez Chumillas y Luis Laorga Gutiérrez, para la residencia de 100 camas; y el primer y segundo premio para el ambulatorio quedó desierto, quedando tercero el arquitecto Ambrosio Arroyo Alonso (16).

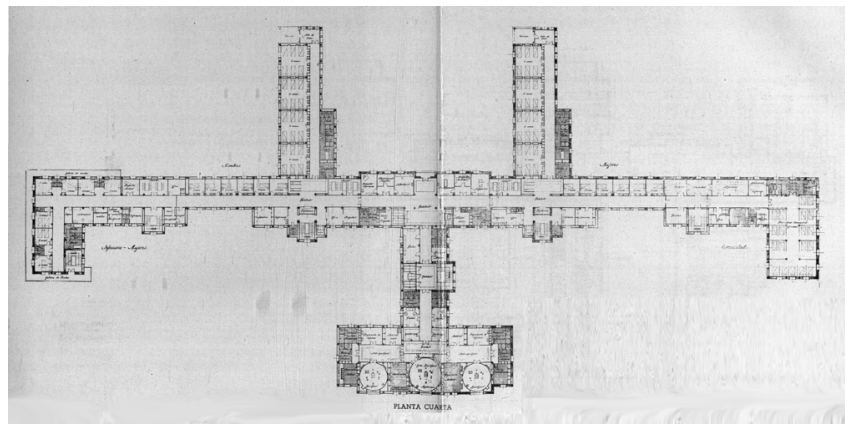

Figura 6. Planta Cuarta del proyecto para la Residencia Sanitaria de 500 camas diseñado por Aurelio Botella Enríquez y Sebastián Vilata Valls, 1946.

Fuente: García Mercadal, F., Botella Enriquez, A., \& Vilata Valls, S. (1947). Concurso de anteproyectos de edificios sanitarios para el seguro de enfermedad: residencia sanitaria para 500 camas : Premios. Revista Nacional de Arquitectura, (62), 50-58.

Los proyectos ganadores para la residencia de 500 y 100 camas, mostraron la línea de diseño hospitalaria iniciada a comienzos del siglo XX: el hospital en bloque, y la organización en forma de «peine» de las plantas de enfermería. Eran diseños de trazado regular y simétrico, con un bloque principal y dos anexos perpendiculares (los «peines») dónde se alojaban las habitaciones para los enfermos. En ambos casos, en los extremos de estos pabellones se situaron terrazas a modo de solárium. Ambas propuestas hacen rememorar los proyectos de hospitales americanos como el Presbyterian Medical Center de Nueva York, diseñado por James Gamble Rogers en 1925, y el hospital español Hospital Clínico San Carlos de Madrid, diseñado por Manuel Sánchez Arcas y Eduardo Torroja en 1928; todos ellos proyectos referentes de la arquitectura hospitalaria moderna del siglo XX, por su capacidad de funcionamiento y de implantación en el lugar a través de una arquitectura de volúmenes simples y rotundos, además de ser pioneros en su tipología.

Poco después de finalizar el concurso, la Comisión de enlace transfirió todas sus competencias al Instituto, y este pasó de nuevo las competencias a una nueva Comisión formada exclusivamente para la ejecución del Plan, que modificó la anterior Orden, la del 19 de febrero de 1945, a través de la Orden del 26 de febrero de 1947, donde se redujo el Plan a 67 residencias y 206 ambulatorios (62 completos y 144 reducidos) (17). Se previó elaborarla en dos etapas: una primera etapa de cinco años donde se construirían 34 residencias y 34 ambulatorios; y una segunda etapa en donde se construirían 33 residencias y 172 ambulatorios.

La finalidad del plan era dotar a las principales capitales de provincia de una asistencia médica completa, con los últimos avances en materia de medicina y diagnóstico. La citada Comisión estuvo a cargo de la organización del Plan hasta el 
año 1950, que, por Decreto, el INP fue reorganizado y creada una Comisaría del Plan Nacional de Instalaciones con una Junta de actuación propia creada el 14 de febrero de 1951. Todos ellos desarrollaron con total independencia las tareas de compra de solares, aprobación de proyectos, ejecución de obras, y adquisición de mobiliario y materiales, tanto para las residencias como para los ambulatorios.

A partir de 1950, comenzaron a inaugurarse los primeros hospitales. Fueron pocos los que abrieron sus puertas en estas fechas; solo se inauguró la Residencia Sanitaria de Valencia y las obras de Mahón, La Coruña, Guadalajara, Logroño, Teruel, Zaragoza, Valencia, Zamora y Madrid tenían las obras avanzadas; el resto estaban en plena construcción (18). Aun así, en 1951 abrieron sus puertas los hospitales de La Coruña, Valencia, Zamora y Madrid, este último, el Hospital Clínico San Carlos fue reconstruido tras los graves daños sufridos durante la Guerra Civil, siguiendo los planos y directrices del arquitecto inicial Manuel Sánchez Arcas.

En 1953 se publicó un folletín propagandístico del Ministerio de Trabajo - Instituto Nacional de Previsión, dónde se puede observar que muchos de los hospitales todavía estaban en fase de construcción. Tras casi una década de la aprobación del Plan, las previsiones iniciales para la primera fase de construcción, estaban lejos de ser cumplidas; y es a partir del año 1955 cuando comenzó la inauguración de la mayor parte de los hospitales de esta primera fase.

Centrándonos en las construcciones hospitalarias y dejando de lado la arquitectura ambulatoria, las construcciones que se inauguraron coparon toda la geografía española, de norte a sur, y de este a oeste. Los mayores hospitales, de más de 500 camas, que se construyeron fueron en Barcelona, con la Residencia Sanitaria Francisco Franco, actual Hospital Vall d'Hebron, que contó el día de su inauguración con 812 camas; en Bilbao con la Residencia Sanitaria Enrique Sotomayor, actual Hospital de Cruces, que contó con 702 camas; en Sevilla con la Residencia Sanitaria García Morato, actual Hospital Universitario Virgen del Rocío con 600 camas; y en Zaragoza, con la Residencia Sanitaria José Antonio, actual Hospital Universitario Miguel Servet que contó con 540 camas.

La Residencia Sanitaria Francisco Franco de Barcelona fue diseñada por el arquitecto Aurelio Botella en 1955. De tipología en bloque, contó con 11 alturas con una planta en forma de «peine» característica. Compuesto por un cuerpo central, se daba acceso a los bloques de enfermería donde estaban las habitaciones que iban de seis pacientes a uno. En la parte trasera del conjunto principal, había un volumen que ocupaba toda la altura del conjunto, donde se alojaban los servicios quirúrgicos, en plantas superiores, y de medicina general, en plantas inferiores. En la planta baja se dispuso las secciones de servicios y administración. La fachada se regía por una composición regular de ladrillo caravista, sin ornamentaciones superficiales, que, sumado al gran tamaño, daba lugar a un conjunto hospitalario imponente y rotundo, con líneas de volumen muy marcadas.

Este mismo arquitecto, Aurelio Botella, en los mismos años, construyó en Granada la Residencia Sanitaria Ruiz de Alda, actual Hospital Virgen de Las Nieves. Con la mitad del número de camas: 446, la curiosidad reside en que ambos edificios son muy similares, se puede decir que la única variación está en el material y color de la fachada, pero el dibujo en planta, su distribución y volumetría son iguales, pudiéndose confundir los planos (figura 7). La similitud entre las distintas residencias sanitarias se debe al método de trabajo llevado a cabo por los arquitectos del Instituto Nacional de Previsión; que, debido a las prisas por proyectar nuevas residencias, tomaban los planos de las que ya estaban construidas, corregían los errores de funcionamiento y planificación detectados, y los desarrollaban en un corto periodo de tiempo.
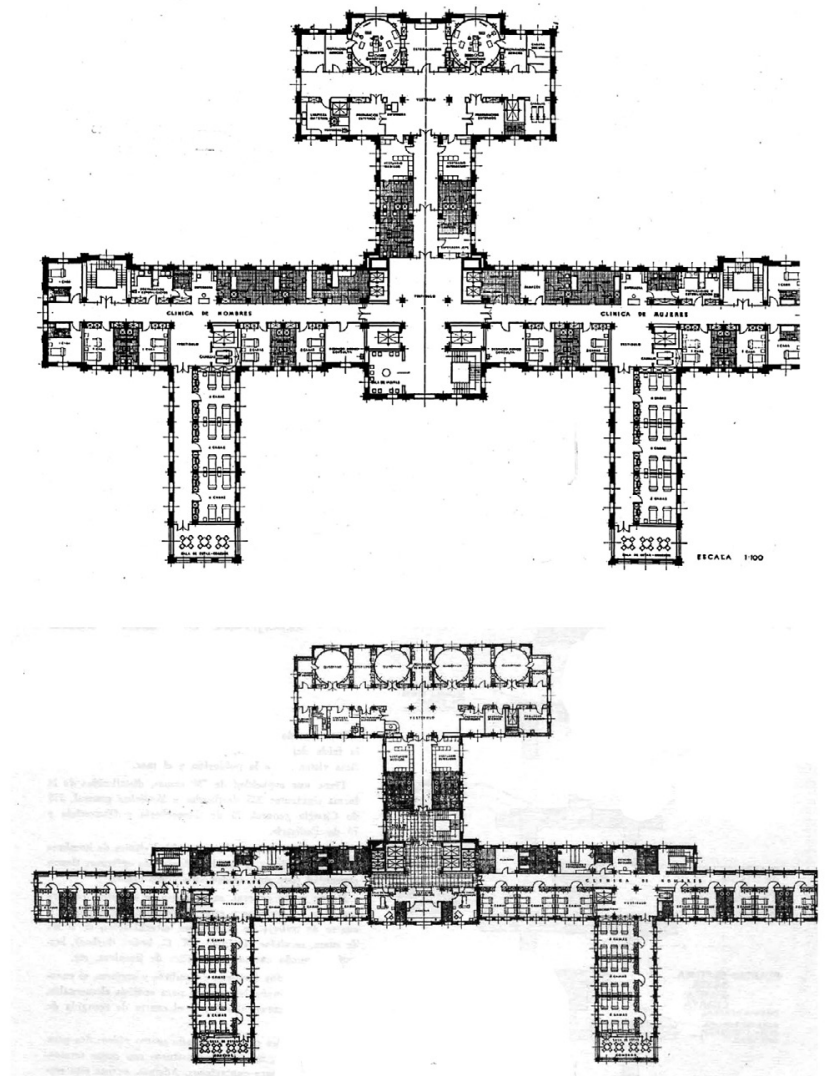

Figura 7. Imagen comparativa de la planta de la Residencia Sanitaria de Barcelona, 1955 (planta superior) y de Granada, 1954 (planta inferior).

Fuentes: Hospital en Granada: arquitecto, Aurelio Botella Enríquez. (1960). Arquitectura, (20), 22-25.

La residencia Sanitaria Enrique Sotomayor (figura 8), fue inaugurada en 1955 con una capacidad de 650 camas. Construida en el municipio de Baracaldo por el arquitecto del instituto Martín José Marcide. La planta se caracteriza por un trazado curvo, concéntrico a la plaza de geometría circular que sirve de entrada y con cinco cuerpos radiales de diferentes alturas. El cuerpo central se elevó para no extender el edificio hacia la parte posterior. Siguiendo el patrón organizativo de la tipología en bloque, los servicios se organizaron en las plantas bajas y sótanos. En cada una de las plantas se dispuso una especialidad, con comunicaciones verticales en el centro y en sus extremos, coincidentes con los volúmenes en perpendicular que sobre salen de su geometría. Respecto a la distribución de las camas, se hizo mejoras respecto a sus precedentes, reduciendo su número a tres y una por habitación. 


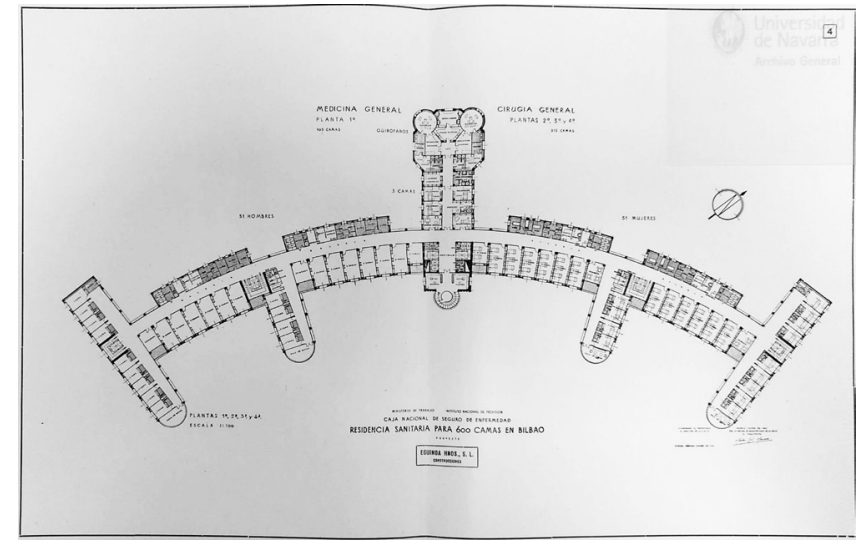

Figura 8. Planta de la Residencia Sanitaria Enrique Sotomayor, 1955.

Fuente: Archivo General de la Universidad de Navarra.

Las Residencias Sanitaras García Morato de Sevilla, de Juan de Zabala y la Residencia Sanitaria José Antonio de Zaragoza (figura 9), de Fernando García Mercadal, ambas inauguradas en 1955, siguieron con el esquema programático de la tipología en bloque: servicios en la planta baja y sótanos, y las especialidades clínicas en plantas superiores con una disposición en planta alargada, comunicadas entre sí por varios núcleos de comunicación verticales. El proyecto de Zaragoza destaca por su gran volumen posterior, de gran longitud y altura, generando un cuerpo de gran esbeltez, como si un gran muro se tratara.
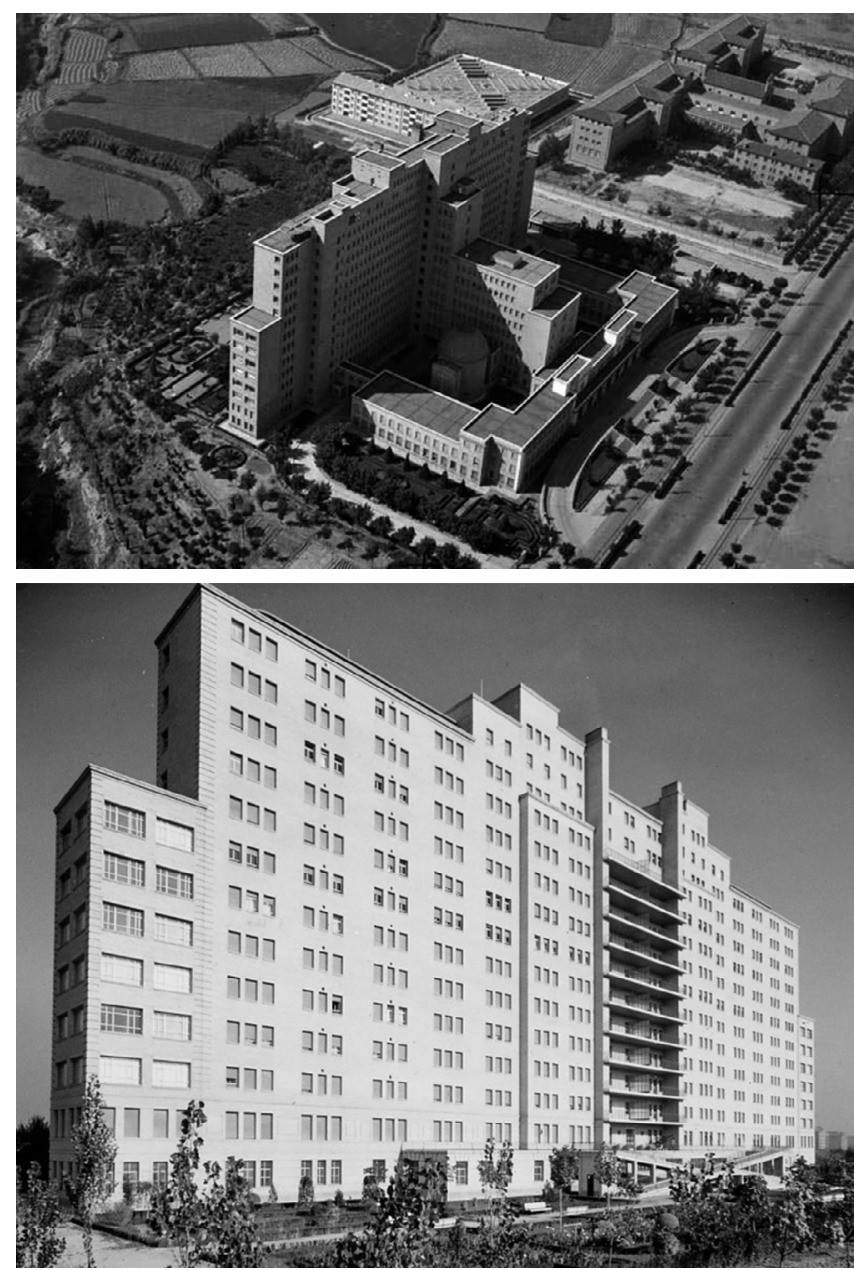

Figura 9. Fotografía aérea y del alzado posterior de la Residencia Sanitaria José Antonio de Zaragoza, 1955
Fuente: Martínez Verón, J. (2016). Hospital Miguel Servet. Retrieved December 31, 2019, from Zaragoza. Arquitectura. Siglo XX

Cabe destacar la Residencia Sanitaria del Almirante Vierna, antiguo Hospital Xeral de Vigo. Del arquitecto Martín José Marcide, fue inaugurado en 1955, con una capacidad de 381 camas. Su tipología difiere de la línea del resto de construcciones, construyéndose en forma de torre. El conjunto estaba compuesto por la suma de dos volúmenes, un cuerpo principal, la torre de 20 plantas, cuya volumetría se escalonaba progresivamente evolucionando de la cruz griega a la cruz latina conforme aumentaba su altura. Y otro volumen bajo, ligeramente curvo, que conformaba el acceso principal desde una amplia escalinata exterior (19).

\subsection{Segunda etapa (1958-1963)}

Hasta 1958 sólo 30, de las 67 residencias sanitarias proyectadas inicialmente, habían sido puestas en funcionamiento. Este número es indicativo de la dificultad que supuso la puesta en práctica del Plan de Instalaciones Sanitarias, fruto de los problemas habidos en cada uno de los tres ejes sobre los que se diseñó el plan: legislativo, financiero y proyectual.

El eje legislativo no supuso un problema significativo, pues el afán del Régimen por construir hospitales era tal, que no escatimó esfuerzos en promover a través de Órdenes ministeriales la creación de nuevas residencias sanitarias y ambulatorio a lo largo y ancho de todo el país. Tal es así, que la orden de ejecución de una nueva remesa de residencias no tardó en llegar tras finalizar el primer periodo de diez años planificado (20). El nuevo plan se basó en establecer dos trienios: el primero de 1958 a 1960 (Orden del 14 de noviembre de 1958) y el segundo de 1961-1963 (Orden del 13 de junio de 1961). En la Orden del 14 de noviembre de 1958, se publicó la orden de construcción de cinco nuevas residencias sanitarias en Cuenca, Salamanca, Santa Cruz de Tenerife, Toledo y Pamplona; y en la Orden del 13 de junio de 1961 se publicó la actuación en las ciudades de Ciudad Real, León, Madrid, Murcia y Orense.

Sin embargo, el eje de la financiación fue un lastre que se arrastró a lo largo de todo el desarrollo del plan y continuó. La lentitud en la ejecución de las obras se puede resumir debido a la falta de financiación pública y a la dependencia financiera de los ingresos derivados de las primas del Seguro Obligatorio de Enfermedad (2) (aunque el sistema de financiación del PNIS era muy complejo y opaco); que derivaron en una situación de déficit presupuestario crónico, una situación difícil de sostener. La vía financiación era muy compleja, y los gastos principales del Plan Nacional de Instalaciones Sanitarias eran elevados. Estos se podían resumir en tres: la compra de solares, los gastos de construcción de las edificaciones y los gastos de montaje de las instalaciones (tabla 2.).

Los proyectos comenzaron una nueva etapa con la entrada de encargos e ideas renovadoras, que comenzaron a modificar la arquitectura hospitalaria que hasta la fecha había construido el Instituto Nacional de Previsión.

El primer paso comenzó con la revisión de lo construido. En 1960, el arquitecto jefe Martín José Marcide publicó en la revista Arquitectura $\mathrm{n}^{\mathrm{0}} 19$, una reflexión acerca de los hospitales construidos en la primera etapa. En este texto resumió las características principales y los errores cometidos, hablando 
Construir 34.00o camas hospitalarias en España. El reto del Plan Nacional de Instalaciones Sanitarias (1942-1982). Build 34,ooo hospital beds in Spain. The challenge of the National Plan of Sanitary Installations (1942-1982).

Tabla 2. Compra de solares, gastos de construcción y montaje de las instituciones del PNIS.

\begin{tabular}{|l|l|}
\hline Años & Importe (pesetas) \\
\hline 1954 & 638.218 .335 \\
\hline 1955 & 332.319 .499 \\
\hline 1956 & 63.920 .143 \\
\hline 1957 & 359.490 .071 \\
\hline Total & 1.393 .948 .048 \\
\hline
\end{tabular}

Fuente: Rull Sabaté, A. (1959). Estudio Estadístico del Seguro de Enfermedad de los años 1954, 1955, 1956 y 1957 (p. 6o). p. 60. Madrid: Archivo IGNESA.

del uso de la tipología en bloque como principal modelo residencial, criticando el problema de circulaciones existente al colocar el eje de comunicaciones en el centro, y de la tendencia hacia la reducción del número de camas por habitación e incorporar el aseo dentro de estas. En esta misma publicación, Marcide, presentó un proyecto de hospital teórico para 300 camas, dedicado en su mayor parte a la hospitalización quirúrgica. Centralizó en una única planta todos los servicios quirúrgicos, solución que más tarde incorporaría en las residencias sanitarias de Segovia, Castellón y Santander. El bloque quirúrgico tenía forma cilíndrica, y sus quirófanos forma ovoidal, tal y como se intuye en las plantas dibujadas.

Y el segundo paso fue la incorporación de arquitectos jóvenes a un grupo reducido ya cerrado desde el comienzo. Uno de ellos fue Fernando Cavestany, quien proyectó la Residencia Sanitaria Virgen de la Vega de Salamanca en 1960 (figura 10). Diseñó un hospital de tipología bloque, compuesto por un cuerpo principal dónde se alojaban los servicios principales como enfermería, quirófanos, consultas, etc.; y unos edificios anexos donde se alojaban las funciones secundarias como los garajes, las instalaciones o servicios. Lo que le hace ser distinto al resto fue su envolvente, un gran muro cortina de vidrio y aluminio, algo inusual hasta la fecha y fuera de la imagen monumental proyectada anteriormente. Esta imagen fue fruto del interés que tenía Cavestany por la arquitectura industrial, sobre todo americana, pues fue invitado por la American Ma-

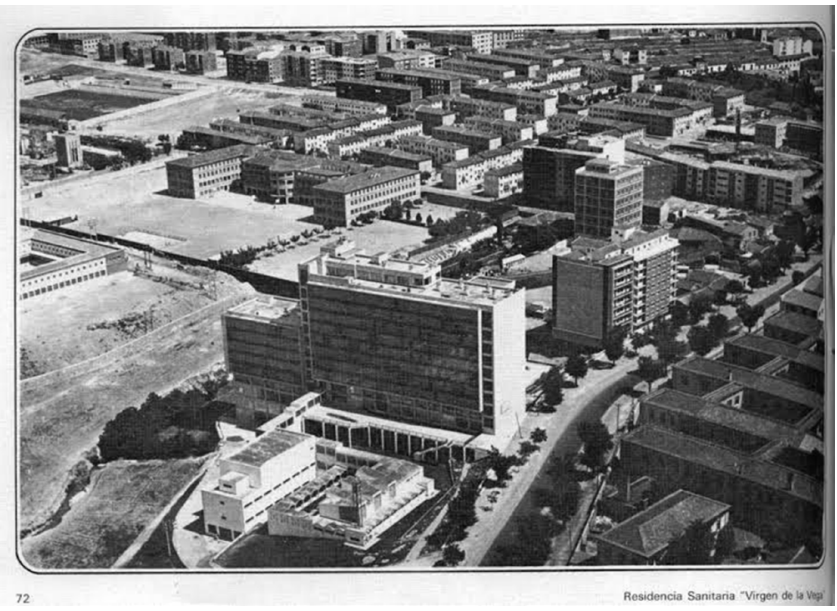

Figura 10. Imagen aérea de la Residencia Sanitaria Virgen de la Vega de Salamanca, de Fernando Cavestany, 1960.

Fuente: Cincuentenario del Hospital Virgen de la Vega de Salamanca. (2015). nagement Associaton para dar una conferencia sobre el tema en 1965 en Nueva York (21).

Los trazados simétricos de los primeros hospitales iban dejando paso a composiciones menos rígidas tomadas de referencias externas de la arquitectura internacional. La Residencia Sanitaria Virgen del Camino de Pamplona (figura 11) diseñada por Fernando García Mercadal en 1960, evita la simetría en planta y la imagen de monumentalidad. Apostó por un cuerpo alargado donde las balconadas corridas a lo largo de toda la fachada tomaron el protagonismo. Además, incorporó los quirófanos ovoides. Años más tarde, en 1967, García Mercadal diseño la Residencia Sanitaria San Jorge de Huesca (figura 12), donde utilizó el mismo esquema de fachada de balcones corridos, habiendo gran similitud entre los dos conjuntos hospitalarios.

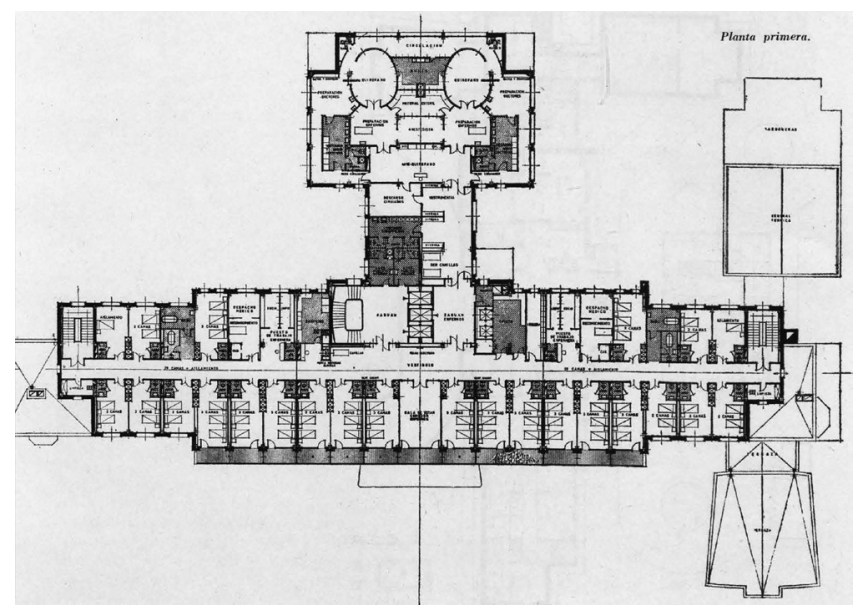

Figura 11. Planta de la Residencia Sanitaria Virgen del Camino de Pamplona, 1960.

Fuente: Hospital en Pamplona: arquitecto, Fernando García Mercadal. (1960). Arquitectura, (20), 41-42.
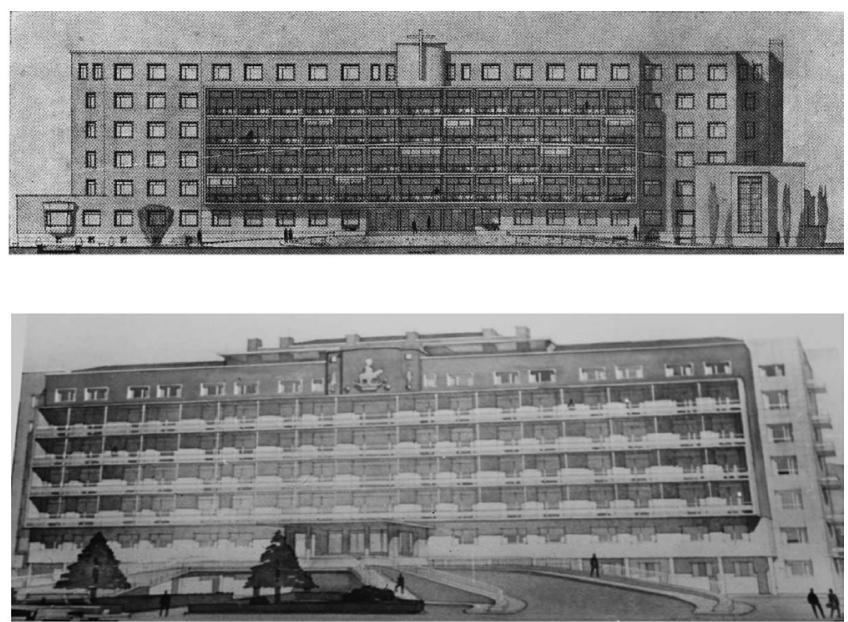

Figura 12. Alzados de la Residencia Sanitaria de Pamplona, 1960 (imagen superior) (1); y de Huesca, 1967 (imagen inferior) (2).

Fuentes: (1) Hospital en Pamplona: arquitecto, Fernando García Mercadal. (1960). Arquitectura, (20), 41-42. (2) Escribano Marquina, E. J. (2017). Arquitectura Hospitalaria del Instituto Nacional de Previsión en Aragón. El Hospital Miguel Servet del arquitecto Fernando García Mercadal. Universidad de Alcalá. 


\subsection{Tercera etapa (1964-1982)}

En 1964 se conmemoró el «25 aniversario de la paz», 25 años del final de la Guerra Civil, marcándose una cima en la carrera hospitalaria española con la construcción de las primeras Ciudades Sanitarias. Se construyeron en Madrid, Barcelona, Valencia, Sevilla, Zaragoza, Bilbao y Oviedo (14). Se renombraron las residencias sanitarias, y así la Residencia Sanitaria García Morato de Sevilla, pasó a llamarse Ciudad Sanitaria Virgen del Rocío; y la Residencia Sanitaria Lorenzo Ramírez de Valencia se llamó Ciudad Sanitaria de La Fe.

La función de las residencias sanitarias era la asistencia quirúrgica, pero se necesitaba más, dar una atención ampliada con la incorporación de servicios de atención pediátrica o traumatológica. Las ciudades españolas habían experimentado por efecto del éxodo rural un crecimiento considerable, y el INP no había conseguido incrementar en consonancia las dotaciones. Por tanto, la idea fue la construcción al borde de las ciudades un conjunto de edificios independientes en función de las necesidades, pero integrados en un sistema de infraestructuras urbanas comunes. De alguna manera recuerdan al esquema organizativo de la tipología pabellonar del siglo pasado, en donde a cada edificio se le otorgaba una función propia.

La Ciudad Sanitaria La Paz de Madrid resumía toda la política asistencial trazada por el gobierno. Fue construida por el arquitecto Martín José Marcide en 1964, y estaba compuesta por una residencia sanitaria, un ambulatorio, una residencia maternal, un hospital infantil (el primero creado por la Seguridad Social) y un centro de rehabilitación y recuperación de inválidos. En la redacción del proyecto los autores desarrollaron su propuesta presidida por tres conceptos fundamentales, según definieron ellos mismos en su memoria técnica: claridad, flexibilidad y concentración de espacio y volúmenes. La planta básica se resolvió con una cruz de brazos desiguales con conexiones entre sí en sus distintos recorridos horizontales y un núcleo central de circulaciones verticales (22). En el conjunto destacaba la torre del edificio de maternidad, de planta circular, forma que ya anticipó Marcide en su hospital ideal para 300 camas.

Se construyó a gran velocidad, con el fin de atenuar el gasto público. Las obras se iniciaron incluso antes de que los proyectos estuviesen terminados, lo cual obligaba a tener que adaptar éstos a lo que ya estaba construido. Con esta forma de proceder, se consiguió batir todos los récords de tiempo a escala internacional, pues el promedio europeo entre planificación y construcción era de diez años, y en España se acortó a poco más de dos. Además, se continuó con la «tradición» de utilizar un mismo proyecto en otra ciudad, así podemos ver nuevamente la similitud entre el edificio de la maternidad de la Ciudad Sanitaria de la Paz y de la del Vall de Hebrón de Barcelona.

Con la llegada de la democracia en 1977 y la Constitución en 1978, en 1979 desapareció el Instituto Nacional de Previsión (INP), sustituido por el INSALUD, el IMSERSO y el INSS, pasando la gestión y administración de los servicios sanitarios al sistema de Seguridad Social y el traspaso de competencias a las autonomías. En 1982, la Oficina de Proyectos del Insti- tuto de Salud se disolvió, poniendo punto y final al Plan de Instalaciones Sanitarias (14).

\section{CONCLUSIONES}

Tras lo expuesto se puede observar como la transformación tipológica del hospital en España ha estado fuertemente marcada por el devenir político-social del país y por la arquitectura internacional que se estaba construyendo, pudiendo estructurar su evolución en dos periodos fundamentales: antes y después de la Guerra Civil (1936-1939).

En los inicios del siglo XX, primer periodo, podemos observar la asimilación de las primeras influencias de la arquitectura hospitalaria en bloque proveniente de Estados Unidos, con una nueva forma de entender el funcionamiento del hospital y su organización basadas en la verticalidad. El hospital pasó a ser un elemento funcional, un instrumento de trabajo útil basado en la superposición de espacios y contenedor de las nuevas tecnologías.

El segundo periodo estuvo marcado por la creación y puesta en marcha del Plan Nacional de Instalaciones Sanitarias. De la necesidad objetiva de falta de hospitales y de la necesidad subjetiva del intento del gobierno por dar una imagen de modernidad y preocupación por la sociedad, nació el Plan. Fue un proyecto de grandes dimensiones, donde el ímpetu por construir lo más rápido posible trajo consigo un lado positivo y otro negativo. La parte negativa fue la pérdida de oportunidad de actualizar las bases de los proyectos, haciendo copias casi exactas unos de otros, sin profundizar en el diseño, lo que trajo perder la línea que estaban trazando los hospitales internacionales más avanzados. Sin embargo, la parte positiva fue la posibilidad de implementar un modelo ya construido y de resolver los problemas.

A todo ello hay que sumarle también las dificultades económicas que supuso su desarrollo, pues un proyecto de tal envergadura requiere de un sistema de financiación fuerte, capaz de soportar las importantes cargas económicas que requiere hacer un edificio hospitalario, y no fue el caso. La guerra dejó a un país sumido en una profunda crisis económica. Esto hizo que los plazos previstos no se cumplieran y que muchos de los proyectos se quedaran en los cajones de las mesas esperando poder ser ejecutados, lo que trajo que el Plan se dilatara en el tiempo, y como es lógico, un proyecto que tarda casi diez años en ser ejecutado, lo hace desfasado.

Pero a pesar de los errores, hay que destacar que, gracias a la elaboración y puesta en marcha del Plan, se creó una red de hospitales por toda la geografía española, y que muchos ellos, a pesar de poder tener fallos funcionales, son ejemplo de la llegada de una nueva arquitectura moderna, lejos de los historicismos y de la arquitectura monumentalista que promovía el régimen. Pues ejemplos como el Hospital Clínico San Carlos de Madrid, el actual Hospital de Cruces (antigua Residencia Sanitaria Enrique Sotomayor) o el Hospital de Salamanca (antigua Residencia Sanitaria Virgen de la Vega) son soplos de modernidad dentro de un periodo de ataduras y censura, que todavía hoy siguen en funcionamiento y son parte de la actual red de hospitales. 


\section{REFERENCIAS}

(1) Sobrino Simal, V. J. (1996). Arquitectura industrial en España (1830-1990).

(2) Pons Pons, J., \& Vilar Rodíguez, M. (2015). El Seguro de Salud Privado y Público en España: Su análisis en perspectiva histórica (1880-2013). Retrieved from https://books.google.es/books?id=Rgv-DAAAQBAJ

(3) de San Antonio Gómez, J. C. (2006). La influencia americana en la arquitectura hospitalaria en la España de los años treinta. In La arquitectura norteamericana, motor y espejo de la arquitectura moderna española en el arranque de la modernidad (1940-1965) (1st ed., pp. 217-226). Pamplona: T6 Ediciones.

(4) Campos Marín, R. (2009). El desarrollo de la salud pública en España durante el siglo XX. Eidon, (32), $67-73$.

(5) Isasi, J., Paniagua, J. L., \& Pieltain, A. (2000). Hospitales. La arquitectura del Insalud 1986-200o (Insalud, Ed.). Madrid.

(6) Lorente de Diego, A., \& Martín Gómez, C. (2017). Evolución funcional y tipológica de los hospitales desde la medicina racional griega hasta la ruptura tecnológica del siglo XX. El hospital como reflejo de las influencias médicas, sociales y tecnológicas. In R. S.L. (Ed.), Actas I Congreso Iberoamericano Redfundamentos: experiencias y métodos de investigación. Madrid.

(7) Iglesias Picazo, P. (2011). La habitación del enfermo. Ciencia y arquitectura en los hospitales del Movimiento Moderno (F. C. de Arquitectos, Ed.). Barcelona: arquia/tesis.

(8) Guerras, J. J. S. (2003). Verticalidad versus horizontalidad. historia de la construcción de hospitales en el siglo xx. Informes de La Construcción, 55, 13.

(9) Sanchez Arcas, M. (1929). La Central médica de Nueva York. Arqutiectura, 119, 141-145.

(10) Sánchez Arcas, M., \& Luis, L. (1930). Proyecto de Hospital provincial para Logroño. Arquitectura, (131), 67-75.

(11) HOSPITAL CLÍNICO UNIVERSITARIO SAN CECILIO. (n.d.). Retrieved November 9, 2019, from Docomomo website: http://docomomoiberico.com/index.php?option=com_k2\&view=item\&id=21:hospital-clinico-universitario-san-cecilio\&Itemid $=55 \& l a n g=e s$

(12) de, A. F., \& Domingo, A. (1934). Anteproyecto de Nuevo Hospital de San Sebastián. Arquitectura, (177), 3-11.

(13) de la Quintana López, P., \& Espinosa Ferrándiz, J. (1944). Seguro de enfermedad : estudio para un plan general de instalaciones de asistencia médica. Madrid: Publicaciones del Instituto Nacional de Previsión.

(14) ÁIvarez-Arenas, A. P. (2003). Los hospitales de Franco : la versión autóctona de una arquitectura moderna (Universidad Politécnica de Madrid - Escuela Técnica Superiro de Arquitectura). Retrieved from http://oa.upm.es/4331/

(15) de Garay, E. (1947). Los hospitales en Estados Unidos. Notas de un viaje de estudios (P. del I. N. de Previsión, Ed.). Madrid.

(16) García Mercadal, F., Botella Enriquez, A., \& Vilata Valls, S. (1947). Concurso de anteproyectos de edificios sanitarios para el seguro de enfermedad: residencia sanitaria para 500 camas : Premios. Revista Nacional de Arquitectura, (62), $50-58$.

(17) INP, P. del (Ed.). (1953). Plan nacional de instalaciones sanitarias del seguro obligatorio de enfermedad. Retrieved from http://bvingesa.mspsi.es/bvingesa/es/catalogo_imagenes/grupo.cmd?path=1002142

(18) Pons-Pons, J., \& Vilar-Rodríguez, M. (2018). Un siglo de hospitales entre lo público y lo privado, 1886-1986. Madrid: Marcial Pons Ediciones de Historia, S.A.

(19) Llamazares Castro, J. (2003). Rehabilitación de fachadas del Hospital Xeral Cíes en Vigo. RE, (33), 70. Retrieved from http://hdl.handle.net/10171/17535

(20) Trabajo, M. de, \& Previsión, I. N. de. (1959). Plan Nacional de Instalaciones Sanitar. Madrid.

(21) Cavestany Milans del Bosch, M. (n.d.). Fernando Cavestany Pardo-Valcarce. Retrieved December 31, 2019, from Real Academia de la Historia website: http://dbe.rah.es/biografias/39826/fernando-cavestany-pardo-valcarce

(22) José, M. M. (1965). Residencia Sanitaria de la Seguriad Social La Paz. Informes de La Construcción, 17(167), 33-47. Retrieved from http://informesdelaconstruccion.revistas.csic.es/index.php/informesdelaconstruccion/article/down$\operatorname{load} / 4436 / 5130$ 